\title{
Cognitive Function Dynamics During Folate Augmented Therapy in Patients with Schizophrenia Carrying MTHFR677C>T Gene Polymorphism: a Pillot Study
}

DOI: 10.17691/stm2015.7.4.20

Received June 23, 2015

T.V. Zhilyaeva, MD, PhD, Tutor, Department of Psychiatry and Medical Psychology;

A.V. Sergeyeva, MD, PhD, Senior Tutor, Department of Epidemiology; Head of Problem Scientific Laboratory of PCR-Investigations, Research Institute of Preventive Medicine;

L.N. Kasimova, MD, DSc., Professor, Head of the Department of Psychiatry and Medical Psychology;

A.S. Blagonravova, MD, DSc, Professor, Department of Epidemiology; Director of Research Institute of Preventive Medicine

Nyzhny Novgorod State Medical Academy, 10/1 Minin and Pozharsky Square, Nyzhny Novgorod, 603950, Russian Federation

The aim of the investigation is to study the carriage of the alleles of folate cycle MTHFR677C>T polymorphism and to assess cognitive function dynamics in the course of folate augmented antipsychotic therapy in schizophrenic patients carrying defective MTHFR677T allele.

Materials and Methods. 129 patients with schizophrenia underwent molecular diagnosing of $677 \mathrm{C}>\mathrm{T}$ polymorphism in MTHFR folate metabolism gene using PCR method with allele-specific primers and the following on-line detection. Of them 18 patients carrying defective T allele received antipsychotic therapy augmented by folates. The patients were tested with a standard battery of cognitive tests before and after augmentation. Patients of the control group $(n=13)$ comparable with the main one in gender, age, psychic condition were subject to the same procedures, but did not receive folates. The level of plasma homocysteine was determined in 7 patients.

Results. Carriage of defective T allele of MTHFR677C >T polymorphism was found in more than half the examined patients, more often than in the general population, though being not statistically significant for such a quantity of observations. Improvement of cognitive functioning was noted in those having defective $T$ allele and receiving folates: short-term auditory memory $(p<0.05)$, phonetic verbal fluency $(p<0.05)$, problemsolving behavior $(p<0.05)$, visual-motor coordination $(p<0.01)$, rigidity $(p<0.05)$, motor velocity $(p<0.05)$, working memory, attention selectivity and distribution $(p<0.05)$. The cognitive function dynamics was more pronounced in the main group than in the control one, however in some patients exacerbation of productive symptoms was observed. Augmentation by folates was most effective in patients with homozygous carriage of the defective $T$ allele and hyperhomocysteinemia before intervention.

Conclusion. Augmentation of antipsychotic therapy by folates may appear to be a promising strategy for improving cognitive functions in patient with schizophrenia having folate metabolism impairments, which requires personified approach with biomarker study before intervention.

Key words: schizophrenia; MTHFR677C>T polymorphisms; hyperhomocysteinemia; folates; folate metabolism impairment.

Patients with schizophrenia have been noted to have various disorders of one-carbon metabolism more often than the general population: decrease of plasma folate and erythrocyte level, hyperhomocysteinemia (an integral marker of folate metabolism impairment), carriage of different genetic polymorphisms of folate cycle $[1,2]$. The polymorphism $677 \mathrm{C}>\mathrm{t}$ of MTHFR gene has been rather well studied: the defective T allele (unlike $C$ allele) reduces the efficiency of one of the enzymes of the folate cycle - methylenetetrahydrofolate reductase (MTHFR) - up to $60 \%$ of the norm. This makes the carrier of the defective allele sensitive to the folate deficiency and contributes to hyperhomocysteinemia development, which, in its turn, results in a number of pathological chemical reactions at the tissue and cell level. Among the people with schizophrenia carriage of MTHFR677T allele occurs more often than in the general population, but some authors do not confirm these data. Thus, the database of the Association of Genetic Polymorphisms with Schizophrenia [www. szgene.org/geneoverview.asp?geneid=4] contains 22 investigations on the association of MTHFR677C > T gene polymorphism with schizophrenia, published in the world literature by the present time, in half of which statistically significant association of $T$ allele carriage with this disease was found. However, findings of other 11 studies are either doubtful or negative. Notably, that carriage of MTHFR677C $>$ T gene polymorphisms is essentially different in the representatives of various regions and nationalities (Table 1), which brings some difficulties in the study of association of these polymorphisms with some diseases, including schizophrenia.

Nevertheless, Muntjewerff et al. [1] having combined in the meta-analysis the data of several investigations,

For contacts: Zhilyaeva Tatiyana Vladimirovna, e-mail: bizet@inbox.ru 
Table 1

Distribution of alleles of MTHFR677C $>$ T polymorphism among the population of some Russian regions and foreign countries

\begin{tabular}{lcl}
\hline \multicolumn{1}{c}{ Region } & T:C frequency ratio & \multicolumn{1}{c}{ Authors } \\
\hline Moscow region, Moscow & $0.296: 0.704$ & Babunova [3]; Kalashnikova, Kokarovtseva [4] \\
\hline Rostov on Don & $0.32: 0.68$ & Miktadova et al. [5] \\
\hline Indonesia & $0.02: 0.98$ & Botto, Yang [6] \\
\hline China & $0.38: 0.62$ & Leclerc et al. [7] \\
\hline USA & $0.55: 0.45$ & Chowdary et al. [8] \\
\hline
\end{tabular}

Table 2

Results of investigations on augmentation of schizophrenic therapy by folates

\begin{tabular}{llll}
\hline \multicolumn{1}{c}{ Authors } & $\begin{array}{c}\text { Time and volume } \\
\text { of observations }\end{array}$ & \multicolumn{1}{c}{ Preparation } & \multicolumn{1}{c}{ Results } \\
\hline Godfrey et al. [13] & 6 months $(n=41)$ & Methylfolate, $15 \mathrm{mg}$ & $\begin{array}{l}\text { Acceleration of clinical and social recovery } \\
\text { Hill et al. [14] }\end{array}$ \\
\hline Roffman et al. [15] & 4 months $(n=28)$ & Folates, $2 \mathrm{mg}$ & $\begin{array}{l}\text { Significant improvement of negative symptoms in carriers } \\
\text { of T allele of MTHFR gene }\end{array}$ \\
\hline Levine et al. [16] & 3 months $(n=42)$ & Folic acid, vitamin $B_{12}$ & $\begin{array}{l}\text { Significant improvement of negative symptoms in carriers } \\
\text { of T allele of MTHFR gene }\end{array}$ \\
\hline
\end{tabular}

demonstrated, that in carriers of TT genotype of MTHFR the likelihood of schizophrenia is $36 \%$ higher than in CC genotype carriers. Peerbooms et al. [9] also showed in their meta-analysis, that TT genotype is encountered significantly more often in schizophrenia than in the general population. According to some publications $[10,11]$, MTHFR677C $>$ T polymorphism is included into the list of 16 genetic risk factors for schizophrenia and of 4 genes with "a strong degree of epidemiological probability" in relation to schizophrenia.

Causal relationships of folate metabolism impairment in the course of schizophrenia development are being actively studied. Of practical interest is the possibility to reduce the risk of the disease by normalizing folate metabolism prior the illness, and to influence the course of the existing disorder. Roffman et al. [12] supposed, that the effect of $T$ allele on the negative schizophrenia symptoms is realized only in case of deficiency of folates in plasma, and this explains the usefulness of augmentation by folates in these patients.

By the present time preliminary data have been obtained in some works (Table 2) on the ability of folates to improve preferably the negative symptoms of schizophrenia without any positive influence upon the productive symptoms.

Nevertheless, the possibility of cognitive symptom correction by folates (important for rehabilitation of patients and their quality of life) was not studied so far. There are several similar investigations of the patients with other psychic disorders (not schizophrenia) and healthy volunteers, in some works positive data have been obtained, but the results are controversial [17]. The assessment of cognitive symptoms depends to the least extent on the subjectivism of the investigator, as the majority of tests are performed by the patient without interference of experimenter, and therefore they present special interest for open researches.

However, the results of interventional investigations in relation to the methods of correcting negative and cognitive schizophrenia symptoms are often ambiguous due to the dissimilarity of the total number of schizophrenic patients. At present more and more data give evidence in favor of the fact, that schizophrenia is not a disease in terms of pathophysiological understanding of the "disease" concept, but a syndrome or even a symptom complex, determined by external (phenotypic) signs, similar in different patients, the internal (genetic, morphological, neurochemical, and others) mechanisms of these symptom development in various patients being different. In this connection homogenization of the schizophrenic patient samples including biomarkers is one of the most perspective ways of overcoming the problem of understanding this disorder and meets the requirement of the modern personified medicine. In particular, when selecting patients for investigation of the efficacy of augmentation by folates it seems to be reasonable to evaluate preliminarily the impairment of folate metabolism taking into consideration genetic or biochemical markers, because it is the correction of the existing disorders of one-carbon metabolism that 
may be of benefit to the patient. And indeed, Roffman in his interventional study [12] convincingly showed, that negative schizophrenic symptoms in augmentation by folates improve only in those patients, who have defective alleles of genetic polymorphism of folate cycle.

The aim of the investigation was to study the carriage of alleles of folate cycle MTHFR677C $>T$ polymorphism and to assess cognitive function dynamics in the course of folate augmented antipsychotic therapy in schizophrenic patients carrying defective MTHFR677T allele.

Materials and Methods. From September 2014 to April 2015129 patients with the diagnosis of "schizophrenia" underwent molecular-genetic investigations to study the carriage of alleles of MTHFR677C $>$ T genetic polymorphism in all-day and day departments of City Clinical Psychiatric Hospital No.1 in Nizhny Novgorod and in-patient department of Nizhny Novgorod Regional Psychiatric Hospital No.3.

Specimens of patients' blood served as the material for investigations. On-line method of polymerase chain reaction with allele-specific primers was used to determine single nucleotide polymorphism. The procedure was performed in the Problem Scientific Laboratory for PCR-investigations of the Research Institute of Preventive Medicine, Nizhny Novgorod State Medical Academy. Mutation of MTHFR Ala222Val (C677T) was determined by the kits manufactured by Litech (Moscow). Three types of conclusions could be made based on the results: normal homozygote, heterozygote, mutant homozygote. A total number of 258 investigations have been performed.

The diagnosis of schizophrenia (paranoid, catatonic, hebephrenic, simple) was established by the hospital physicians before the beginning of the study and was registered in the initial medical records. The age of the patients varied from 20 to 65 years (61 females, 68 males).

The study complied with the Declaration of Helsinki (the Declaration was passed in Helsinki, Finland, June, 1964, and revised in October, 2000, Edinburg, Scotland) and was performed following approval by the Ethic Committee of Nizhny Novgorod State Medical Academy. Written informed consent was obtained from every patient.

Part of the patients - carriers of the defective allele MTHFR677T ( $n=18$, among which 3 were homozygous and 15 heterozygous) agreed to add folic acid to the administered antipsychotic therapy in the dose of 0.5$1.0 \mathrm{mg} /$ day per os. The dose of folic acid was initially determined as $1 \mathrm{mg} /$ day (maximal prophylactic), but in the process of investigation it became evident, that in some patients intensification of psychotic symptoms was observed when the acid was taken in the given dose, therefore, the folate dose was reduced to $0.5 \mathrm{mg} /$ day for the patients with productive symptoms.

Augmentation was performed after the reduction of acute psychotic symptoms not less than 2 weeks after the admission to the in-patient department (patients' behavior was normalized, they were able to comprehend the tasks of the investigation and give a written informed consent for participation). Prior to administration of folic acid patients underwent a standard battery of cognitive tests: method of "learning of 10 words", Stroop test, tapping-test, tests of verbal semantic and phonetic fluency, letter-number sequencing test, "tower of London" test, Munsterberg test, and "coding" from Wechsler test. The battery of cognitive tests was chosen from the tools, which, according to the data of recent studies, are most reliable and often used for diagnosing cognitive functions in schizophrenia [18].

About 4 weeks after continuous daily intake of folic acid concurrently with antipsychotic therapy testing was repeated. The interval between the first and second testing of cognitive function was not less than 3 weeks since the level of homocysteine during folate intake normalizes in 2-3 weeks; and not more than 5 weeks due to the standard terms of treating schizophrenia in hospitals.

To exclude the influence of the "learning effect" (improved results caused by repeated testing) and other artefacts, control group of patients being in the same conditions but without administration of folic acid was composed $(n=13)$, among which 6 patients were heterozygous carriers of the defective allele, 7 patients with homozygote by allele $\mathrm{C}$. Patients of the control group also had the diagnosis of "schizophrenia", established before the participation in the investigation, stayed in the hospital for about 2 weeks and were tested with the battery of cognitive tests twice with the interval of 3-5 weeks. The main and control groups were comparable in age, sex, duration of the disease and the administered antipsychotic therapy.

Statistical analysis of the results obtained was performed on the basis of MS Excel software using paired two sample t-test for means and Statistica 6.0 using non-parametrical statistical methods.

Results and Discussion. Carriage of MTHFR677T allele was found in 68 patients of $129(52.7 \%)$, among them $10(7.8 \%)$ were homozygous carriers (MTHFR677TT), $58 \quad(45.0 \%)$ heterozygous (MTHFR677CT); the rest $(n=61,47.3 \%)$ were carriers of normal homozygote (MTHFR677CC). The total carriage (homo- and heterozygous) of the defective allele is noted to be more frequent than in the general population (See the Figure), but the number of observations by the present time is not large enough to confirm statistical significance of differences. It is notable, that the portion of heterozygous carriers is large, but at the same time the portion of homozygous carriers of the defective allele is less in the patient sample. It is likely to be connected with early lethality in patients with MTHFR677TT genotype, since in some investigations its carriers have a higher risk of heavy cardiovascular, endocrine and oncologic 


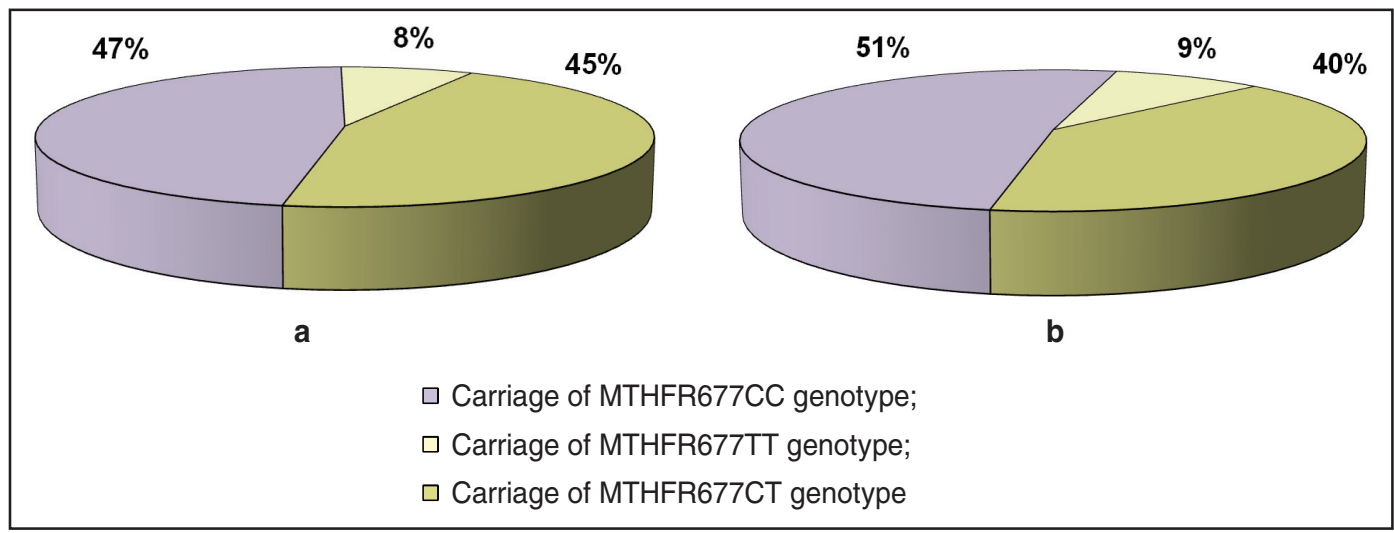

Distribution of MTHFR677C>T genotypes: (a) in the examined sample of patients with schizophrenia; (b) in the general population of Moscow region $[3,4]$

illnesses $[1,19,20]$. It will further require the analysis of $\mathrm{C} / \mathrm{T}$ allele distribution among various age groups and age-related comparison of the sample of patients with schizophrenia with the general population, if there is a greater bulk of observations.

Data on the distribution of MTHFR677C $>$ T genotypes in the general population (See Figure (b)) are presented for Moscow region, since such information in Nizhny Novgorod is not available. The Moscow region is not far from Nizhny Novgorod, however, the population of Moscow is mainly represented by migrants from other regions of the Russian Federation and CIS, which may influence the carriage of genetic alleles, as MTHFR alleles have a non-uniform geographic distribution [3-8]. Therefore, it is necessary to compare the studied sample with the data on Nizhny Novgorod region in order to obtain reliable valid results on the association of the $T$ allele carriage with schizophrenia incidence.

In the main group receiving folates $(n=18)$ statistically significant improvement of various indices was noted: short-term auditory memory (learning of 10 words) $(p<0.05)$, phonetic verbal fluency $(p<0.05)$, problem-

Table 3

Dynamics of cognitive functions in the group receiving folate augmentation and the control group

\begin{tabular}{|c|c|c|}
\hline Index & $\begin{array}{l}\text { Folate } \\
\text { augmentation } \\
(n=18)\end{array}$ & $\begin{array}{l}\text { Control } \\
(n=13)\end{array}$ \\
\hline $\begin{array}{l}\text { Short-term auditory memory } \\
\text { "learning of } 10 \text { words" }\end{array}$ & $\uparrow \uparrow, p<0.05$ & $\uparrow, p>0.05$ \\
\hline Phonetic verbal fluency & $\uparrow \uparrow, p<0.05$ & $\uparrow, p>0.05$ \\
\hline $\begin{array}{l}\text { Problem-solving behavior ("tower } \\
\text { of London") }\end{array}$ & $\uparrow \uparrow, p<0.05$ & $\uparrow, p>0.05$ \\
\hline Visual-motor coordination ("coding") & $\uparrow \uparrow, p<0.01$ & $\uparrow \uparrow, p<0.05$ \\
\hline
\end{tabular}

He re: $\uparrow$ weak, statistically insignificant positive dynamics; $\uparrow \uparrow$ distinct, statistically positive dynamics; $\uparrow \uparrow \uparrow$ marked, statistically highly significant positive dynamics.
Table 4

Dynamics of cognitive functions in the group receiving folate augmentation after exclusion of patients with deteriorated condition and in the control group

\begin{tabular}{|c|c|c|}
\hline Index & $\begin{array}{l}\text { Folate } \\
\text { augmentation } \\
(n=13)\end{array}$ & $\begin{array}{l}\text { Control } \\
(n=12)\end{array}$ \\
\hline Rigidity (Stroop test) & $\uparrow \uparrow, p<0.05$ & $\uparrow, p>0.05$ \\
\hline Motor velocity ("tapping”) & $\uparrow \uparrow, p<0.05$ & $\uparrow, p>0.05$ \\
\hline $\begin{array}{l}\text { Morking memory, attention } \\
\text { distribution and selectivity } \\
\text { ("letter-number sequencing") }\end{array}$ & $\uparrow \uparrow, p<0.05$ & $\uparrow, p>0.05$ \\
\hline $\begin{array}{l}\text { Attention selectivity (Munsterberg } \\
\text { test) }\end{array}$ & $\uparrow \uparrow \uparrow, p<0.01$ & $\uparrow \uparrow, p<0.05$ \\
\hline
\end{tabular}

He re: $\uparrow$ weak, statistically insignificant positive dynamics; $\uparrow \uparrow$ distinct, statistically positive dynamics; $\uparrow \uparrow \uparrow$ marked, statistically highly significant positive dynamics.

solving behavior (tower of London) $(p<0.05)$ and visual-motor coordination ("coding" from Wechsler test) $(p<0.01)$ (Table 3$)$, other cognitive functions also improved with folate intake, but statistically insignificantly (t-test for repeated measurements). In 6 patients psychic condition worsened due to the intensification of the productive symptoms (among them 5 patients from the main group, 1 from the control; all patients were heterozygous carriers of $\mathrm{T}$ allele), which influenced the results of cognitive testing. When these patients were excluded from the analysis, a positive dynamics of such indices as rigidity (Stroop test, $p<0.05$ ), motor velocity ("tapping", $p<0.05$ ), working memory, attention selectivity and distribution (Munsterberg tests, $p<0.01$ ), and letter-number sequencing, $(p<0.05)$ (Table 4$)$ was also statistically significant.

In the control group receiving treatment without folic acid indices of selective attention (Munsterberg test) $(p<0.05)$ and visual-motor coordination ("coding") 
$(p<0.05)$ improved significantly, but short-term auditory memory ("learning of 10 words") and semantic verbal fluency became worse, other cognitive function had a small statistically insignificant tendency to improvement. In this connection improvement of indices measured by the "coding" method and Munsterberg test in the main group could not also be considered the result of folate augmentation, since it may be so that the results in patients of both groups were improving owing to antipsychotic therapy or learning when the test was repeated.

Multidirectional dynamics of cognitive indices within the group of patients, receiving folates, may be caused by heterogeneity of patients in terms of initial disorders in folate metabolism in the sample selected for augmentation. So, a positive dynamics was noted in all three homozegous carriers of the defective $T$ allele in the main group $(n=18)$. But in heterozegous carriers (15 of 18 patient of the main group) cognitive functions were changing in various directions - from significant improvement to significant worsening with aggravation of productive symptoms. In several patients $(n=7)$ integral index of folate metabolism was examined the level of plasma homocysteine: in some patients it appeared to be within the norm, while in others it exceeded the norm essentially (Table 5). It is important to note, that the "norm" of plasma homocysteine - up to $13 \mu \mathrm{mol} / \mathrm{L}$ - recommended by the majority of Russian laboratories became obsolete: according to the WHO recommendations the level of homocysteine below $10 \mu \mathrm{mol} / \mathrm{L}$ may be considered optimal. Patients with more severe hyperhomocysteinemia had a more pronounced positive response to folate therapy. The difference in the homocysteine level within genetically homogenuous groups by MTHFR677C >T polymorphism was likely to be due to the character of interaction of the studied gene with other genetic polymorphisms of the folate cycle, and with the environmental factors (nutritional habits and lifestyle) as well.

Thus, carriage of the defective $T$ allele of MTHFR677C $>T$ polymorphism was revealed in more than half examined patients with schizophrenia - more

Table 5

Homocysteine level in some patients with different MTHFR genotypes

\begin{tabular}{|ccc|}
\hline Patients & $\begin{array}{c}\text { MTHFR } \\
\text { genotype }\end{array}$ & $\begin{array}{c}\text { Homocysteine level } \\
(\boldsymbol{\mu m o l} / \mathbf{L})\end{array}$ \\
\hline Patient 1 & TT & 28 \\
\hline Patient 2 & TT & 166 \\
\hline Patient 3 & CT & 23 \\
\hline Patient 4 & CT & 8.14 \\
\hline Patient 5 & CC & 8.65 \\
\hline Patient 6 & CC & 12.53 \\
\hline Patient 7 & CC & 11.02 \\
\hline
\end{tabular}

often than in the general population, but it is statistically insignificant due to the small number of the available observations. Carriers of defective $\mathrm{T}$ allele receiving folates are noted to improve cognitive functioning more significantly and markedly than in the control group, however, there is a risk of productive symptom aggravation. The predictor of efficacy of folate augmented therapy may be the intensity of hyperhomocyteinemia prior to intervention.

Conclusion. Folate augmented therapy of patients with schizophrenia, carriers of the defective MTHFR677T allele, promotes the improvement of the cognitive symptoms of the disease, especially in those having hyperhomocyteinemia, which suggests the benefit of implementing the given approach into clinical practice.

Study Funding. The work was supported by the funds allocated for research works of the Research Institute of Preventive Medicine of Nizhny Novgorod State Medical Academy "Innovative technologies of monitoring and prophylaxis of current diseases" and research works of the Department of Psychiatry and Medical Psychology of Nizhny Novgorod State Medical Academy "The study of one-carbon metabolism impairment in patients with mental disorders" (State registration No.01201460720).

Conflicts of Interest. The authors declare no conflicts of interest related to this study.

\section{References}

1. Muntjewerff J.W., Kahn R.S., Blom H.J., den Heijer M. Homocysteine, methylenetetrahydrofolate reductase and risk of schizophrenia: a meta-analysis. Mol Psychiatry 2006; 11(2): 143-149, http://dx.doi.org/10.1038/sj.mp.4001746.

2. Zhilyaeva T.V. One-carbon metabolism impairment in schizophrenia. Psikhiatriya $i$ psikhofarmakoterapiya im. P.B. Gannushkina 2012; 14(6): 41-46.

3. Babunova N.B. Izuchenie assotsiatsii genov AGT, AGT1R i MTHFR s serdechno-sosudistymi zabolevaniyami. Dis. ... kand. biol. nauk [The study of AGT, AGT1R and MTHFR gene associations with cardiovascular diseases. PhD Dissertation]. Moscow; 2004.

4. Kalashnikova E.A., Kokarovtseva S.N. Association between congenital thrombophilia and fetal loss in russian population. Meditsinskaya genetika 2005; 4(8): 386-390.

5. Miktadova A.V., Kovalenko K.A., Baronyan E.S., Mashkina E.V., Shkurat T.P. Analysis of alleles frequencies of candidate genes in Rostov-on-Don. Sovremennye problemy nauki i obrazovaniya 2012; 5: 276-285.

6. Botto L.D., Yang Q. 5,10-Methylenetetrahydrofolate reductase gene variants and con congenital anomalies: a HuGE review. Am J Epidemiol 2000; 151(9): 862-877, http://dx.doi. org/10.1093/oxfordjournals.aje.a010290.

7. Leclerc D., Wilson A., Dumas R., Gafuik C., Song D., Watkins D., Heng H.H., Rommens J.M., Scherer S.W., Rosenblatt D.S., Gravel R.A. Cloning and mapping of a cDNA for methionine synthase reductase, a flavoprotein defective in patients with homocystinuria. Proc Natl Acad Sci USA 1998; 95(6): 3059-3064, http://dx.doi.org/10.1073/pnas.95.6.3059.

8. Chowdary D., Streck D., Schwalb M.N., Dermody J.J. High incidence of two methylenetetrahydrofolate reductase 
mutations (C677T and A1298C) in Hispanics. Genet Test 2003; 7(3): 255-257, http://dx.doi.org/10.1089/109065703322537296.

9. Peerbooms O.L., van Os J., Drukker M., Kenis G., Hoogveld L., de Hert M., Delespaul P., van Winkel R., Rutten B.P. Meta-analysis of MTHFR gene variants in schizophrenia, bipolar disorder and unipolar depressive disorder: evidence for a common genetic vulnerability? Brain Behav Immun 2011, 25(8): 1530-1543, http://dx.doi.org/10.1016/j.bbi.2010.12.006.

10. Allen N.C., Bagade S., McQueen M.B., Ioannidis J.P., Kavvoura F.K., Khoury M.J., Tanzi R.E., Bertram L. Systematic meta-analyses and field synopsis of genetic association studies in schizophrenia: the SzGene database. Nat Genet 2008; 40(7): 827-834, http://dx.doi.org/10.1038/ng.171.

11. van den Buuse M. Modeling the positive symptoms of schizophrenia in genetically modified mice: pharmacology and methodology aspects. Schizophr Bull 2010; 36(2): 246-270, http://dx.doi.org/10.1093/schbul/sbp132.

12. Roffman J.L., Brohawn D.G., Nitenson A.Z., Macklin E.A., Smoller J.W., Goff D.C. Genetic variation throughout the folate metabolic pathway influences negative symptom severity in schizophrenia. Schizophr Bull 2011; 39(2): 330-338, http:// dx.doi.org/10.1093/schbul/sbr150.

13. Godfrey P.S., Toone B.K., Carney M.W., Flynn T.G., Bottiglieri T., Laundy M., Chanarin I., Reynolds E.H. Enhancement of recovery from psychiatric illness by methylfolate. Lancet 1990; 336(8712): 392-395, http://dx.doi.org/10.1016/01406736(90)91942-4.

14. Hill M., Shannahan K., Jasinski S., Macklin E.A.,4 Raeke L., Roffman J.L., Goff D.C. Folate supplementation in schizophrenia: a possible role for MTHFR genotype. Schizophr Res 2011;
127(1-3): 41-45, http://dx.doi.org/10.1016/j.schres.2010.12.006.

15. Roffman J.L., Lamberti J.S., Achtyes E., Macklin E.A., Galendez G.C., Raeke L.H., Silverstein N.J., Smoller J.W., Hill M., Goff D.C. Randomized multicenter investigation of folate plus vitamin B12 supplementation in schizophrenia. JAMA Psychiatry 2013; 70(5): 481-489, http://dx.doi.org/10.1001/ jamapsychiatry.2013.900.

16. Levine J., Stahl Z., Sela B.A., Ruderman V., Shumaico O., Babushkin I., Osher Y., Bersudsky Y., Belmaker R.H. Homocysteine-reducing strategies improve symptoms in chronic schizophrenic patients with hyperhomocysteinemia. Biol Psychiatry 2006; 60(3): 265-269, http://dx.doi.org/10.1016/ j.biopsych.2005.10.009.

17. Balk E.M., Raman G., Tatsioni A., Chung M., Lau J., Rosenberg I.H. Vitamin B6, B12, and folic acid supplementation and cognitive function: a systematic review of randomized trials. Arch Intern Med 2007; 167(1): 21-30, http://dx.doi.org/10.1001/ archinte.167.1.21.

18. Sarkisyan G.R., Gurovich I.Ya., Keefe R.S. Normative data for the russian population and standardization of the Brief Assessment of Cognition in Schizophrenia (BACS) Scale. Sotsial'naya i klinicheskaya psikhiatriya 2010; 20(3): 13-19.

19. Fetisova I.N., Dobrolyubov A.S., Lipin M.A., Polyakov A.V. The polymorphism of folate metabolism genes and human diseases. Vestnik novykh meditsinskikh tekhnologiy 2007; X(1): 32-38.

20. Gnezdilova I.V., Akhmadishina L.Z. The study of methylenetetrahydrofolate reductase (MTHFR) gene role in the formation of susceptibility to thrombophilia. Molodoy uchenyy 2010; 1(2-1): 123-126. 